\title{
Computer Science Approach to Information-Like Artifacts as Exemplified by Memes
}

\author{
Sabah Al-Fedaghi \\ Computer Engineering Department \\ Kuwait University \\ Kuwait
}

\begin{abstract}
Providing information can be expanded to include systems that deliver information-like artifacts. They provide such "things" as advertisements, propaganda pieces, and meme artifacts. Memes are the subject of extensive intellectual debate in science and popular culture because it is claimed that parallels can be drawn between theories of cultural evolution manifested in memes, and theories of biological evolution. Memes are described as self-reproducing mental structures, intangible entities transmitted from mind to mind, verbally or by repeated actions and/or imitation. The problem is that researchers describe memes in terms of English-language text or ad hoc diagrams. This paper considers the problem that the field of memetics lacks a uniform language for examining diverse conceptualizations of memes. The paper presents a unifying diagrammatic representation used in computer science, in which all types of "claimed" memes can be expressed and their general characteristics observed. Several examples from the literature on memes are recast in terms of this representation. The results point to the capability of the proposed depiction to express various types of memes.
\end{abstract}

Keywords-information; cultural evolution; mental structures; memes; memetics; conceptual modeling; diagrammatic representation

\section{INTRODUCTION}

Information Systems attempts to provide the business client with information that maximizes its effectiveness [1]. Going further, it is possible to remove the restriction of providing information, to include systems that provide clientele with information-like artifacts that maximize their effectiveness. They include systems that provide such "things" as advertisements, propaganda pieces, and meme artifacts. Memes, the topic of this paper, are of special importance in this context, expressed as follows:

We are potentially facing what is termed a General Purpose Technology or Disruptive Innovation. If this prediction proves right, the world will be ripe for a new version of James Brown's “It's a Man's Man's Man's World” to be titled “It's a Memes' Memes' Memes' World' [2]. (Italics added)

This paper tries to strengthen the notion of meme by proposing a schematic representation of transmission of memes. Memes (cultural artifacts) are interesting "flow things" because of their complex conceptual relationships with "life things" or genes.

Few scientific terms introduced into scientific and popular vernacular have enjoyed the impact on intellectual debate as has the term "meme."... The meme concept has generated recent excitement precisely because it seems to offer hope of providing something that other theories of social and semiotic processes have not succeeded in providing. [3].

Memes are usually described as units of cultural flow that may change in terms of host state (temporal/special), population (growth through replication), and variety (differences). The following paragraphs highlight some of the literature pertaining to memetics: "the theoretical and empirical science that studies the replication, spread and evolution of memes" [4]. The focus here is on more description of the notion of meme, the life cycles of memes, and modeling of memes. Modeling in this context refers to development of an abstract representation of a meme life cycle through diagrammatic representation of its flow from birth to its destination, including its effects on its hosts.

\section{A. About Memes}

\section{According to Dawkins [5],}

We need a name ... that conveys the idea of a unit of cultural transmission, ... I want a monosyllable that sounds a bit like "gene"... it could alternatively be thought of as being related to "memory"... Just as genes propagate themselves in the gene pool by leaping from body to body via sperms or eggs, so memes propagate themselves in the meme pool by leaping from brain to brain via a process which, in the broad sense, can be called imitation. (p. 192)

Memes are also described as self-reproducing mental structures, intangible entities, transmitted from mind to mind, either verbally, with actions, music, graffiti, leaflets, television broadcasts, or by repeated actions and/or imitation. Hence, they have been viewed as "a base for constructing an illustrative model of social and cultural behavior raising questions about how memes are generated, received, transmitted, replicated, and re-transmitted" [6]. Memes "influence ideas, ideas influence and form beliefs ..., eventually producing actions" [6].

The replication and transmission of memes in culture have been described in analogy to biological genes [7]. A combined group of memes is called a memeplex. A central concept in the meme-gene analogy is replication. A replicator is first defined as a unit that has largely the same structure before and after a process of copying or replication is completed. Some errors may be made in the process, but too many errors are not 
allowed for a copying process to count as a replication process [8].

The second essential characteristic [of a meme] is that it is part of a lineage. Genes are called replicators because they are copied over and over. Over and over means that a replicator is first copied from a mould, then in a next replication event it is used as a mould itself, from which a new copy is produced, that again will function as a mould in the next event, and so on [8].

The media by which a meme flows (e.g., journals, radio stations) affects its life cycle. Computer networks currently play a major role as a meme environment. An Internet meme is a meme that spreads online, e.g., via online social networks. It refers to the propagation of content items such as jokes, videos, and websites from one person to another via the Internet [9].

\section{B. Meme Life Cycle}

According to Heylighen [10], memes go through a fourstage life cycle: assimilation, transmission, expression, and retention. Assimilation happens when a meme arrives at a new host and is accepted and processed. At this stage, the "meme is very vulnerable to misunderstanding" [11]. Expression is when "a meme must emerge from its storage as memory pattern and enter into a physical shape that can be perceived by others" [10]. Transmission is when the meme is presented through some form of media. Retention is when the host "memorizes" the meme.

For Bjarneskans, Grønnevik, and Sandberg [12], the meme life cycle is similar to that of parasites. It includes transmission, decoding, infection, and coding phases. Encoding of the meme happens in the transmission phase, when the meme is coded in a vector such as spoken message, text, image, e-mail, or observed behavior. It is "some kind of information-carrying medium."

When a potential host decodes the meme ... the meme may become active and infect the person, who becomes a new host (the infection phase). At some point the meme is encoded in a suitable vector (not necessarily the same medium it was originally decoded from) and can be spread to infect new hosts [12].

\section{Modeling and Representation}

The brief, general description of memes and their life cycle presented above establishes this paper's topic and its associated discourse. This subsection closes in on the center of interest: modeling, and in particular producing a conceptual representation of the flow of memes and learning the significance of that flow.

Much of the published research draws parallels between theories of cultural evolution and those of biological evolution [13]. A typical dictionary definition of evolution describes it as a process of continuous change of something into a different and usually more complex or new form. Change is a fundamental notion with a long history. Heraclitus [14], a preSocratic Greek philosopher, observed that everything changes and nothing remains still [3]. He described this change as a flow of things, declaring that "everything flows." Flow can be observed everywhere, especially in nature and in social ontology, e.g., flows of water, electricity, gas, money, materials, memes, and so on.

Such a view aims at capturing a real-world process, specified in text or diagram form or written in a formal language. "The resulting depiction expresses a process model, a process template, process metadata,... A process definition normally comprises a number of discrete activity steps, with associated computer and/or human operations and rules governing the progression of the process through the various activity steps" [15]. In this perspective, modeling provides a framework for organizing and enabling future exploration [16]. In the context of the current topic, it is used to observe the path of memes moving through hosts in order to develop a broader theoretical basis for examining the changes that occur in memes over time [16].

For example, Husted [16] proposes a multilevel model for cultural meme transference based on the behavior of the ocean ecosystem. "The ocean works as a model because organic materials move through the ocean in the same way that cultural objects move through society" [16]. Communities of memes can be compared with a coral reef within a larger ocean ecosystem; similarly, the Internet is an adaptive entity consisting of many cooperative communities of organisms [16]. Coral reef production of biodiversity is analogous to the forum cultures that produce the largest number of memes on the Internet.

A coral reef represents a meme-producing core society. Different inhabitants are specified in the model, e.g., lurking users who do not participate in the communities they are observing, along with core memes that attack inhabitants of other levels.

\section{Problem and Solution}

This paper addresses the problem of lack of a uniform model for examining diverse conceptualizations of memes. For example, a question answered in the context of this problem: How to represent such diverse things as graffiti and strategy, which have been claimed to be memes, in a uniform way so their common characteristics (e.g., evolution, spreading, multiplicity, ...) can be compared? The paper presents a unifying conceptual representation in which all types of "claimed" memes can be expressed and their general characteristics observed.

For the sake of a self-contained paper, we next review the model on which our representation of the flow of memes will be built. After that, in the section titled "Schematizing Memes," a meme is described as a special type of "flow thing" by use of schemata (patterns of streams of flow) based on this model.

The section models changes in a meme through flow across hosts by drawing schemata of flows, including five stages in the life cycles of each host. The fourth section, titled "Applying FM to Sample Meme Systems," applies this representation of meme flow in three areas: organizational strategy, humancentered models of processes, and agents' decisions with regard to meme adoption and transmission. 


\section{FLOWTHING MODEL}

The Flowthing Model (FM) [17-22] represents some segment of reality as a web of interrelated flows that cross boundaries of intersecting and nested spheres. In the context of memes, every host is represented by a sphere with a hierarchical structure. Ingredients in a flow include flowthings (things that flow, e.g., memes), and their systems (flowsystems): a structure of flow comprising at most six stages (see Fig. 1). A "thing" is defined as a flowthing: what is created, released, transferred, arrived, accepted, and processed while flowing within and among spheres. It has a permanent identity but impermanent form, e.g., the same memes translated into different languages. A flowsystem constrains the trajectory of flow of flowthings. A particular flowsystem is the space/time context for happenings (e.g., received, released) and existence of flowthings. From the perspective of flowthings, the flowsystem is formed from six discontinuities: being created, being released, being transferred, being arrived, being accepted, and being processed.

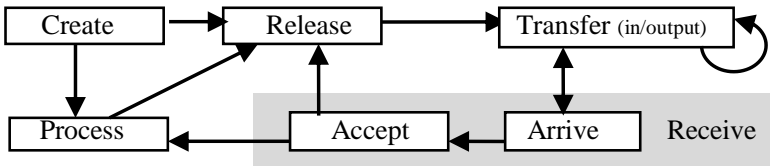

Fig. 1. Flowsystem

Flows connect six states (also called stages) that are exclusive for flowthings; i.e., a flowthing can be in only one of these six states at a time: transfer, process, creation, release, arrival, or acceptance, as shown in Fig. 1, analogous to water being in one of three states in Earth's atmosphere: solid, liquid, or gas. A state here is a "transmigration field" of the flowthing that is created, processed, and released, transferred, arrives, and is accepted (or is simply received, combining arrived and accepted into one state). In Fig. 1, we assume irreversibility of flow, e.g., released flowthings flow only to Transfer.

The exclusiveness of FM states (i.e., a flowthing cannot be in two states simultaneously) indicates synchronized change of the flowthing, e.g., a flowthing cannot be changed in form and sphere simultaneously. This is a basic systematic property of flowthings. Note the generality of the notion of flow in FM. For example, creation of a flowthing is a flow (from nonexistence, i.e., not currently existing in the system, to existence, i.e., appearance in the system).

Initialization, stopping, and continuing of flows occur through triggering: a control mechanism. It is the only linkage among elements in FM description besides flow and is indicated by dashed arrows. Synchronizations (e.g., join/fork) and logic notions (e.g., and/or) can be superimposed over the basic FM depiction. Note that these mechanisms can be modeled as flowsystems.

\section{SCHEMATIZING MEMES}

The thesis of this paper is that a meme is a special type of flowthing with a distinctive schema or pattern of streams of flow. Several works have been published classifying memes as a general category. Deacon [3], in semiotics, suggests that "Memes are signs, or more accurately, sign vehicles... They are ... concrete things, or events, or processes."
In FM, a general conceptualization of a meme (see Fig. 2) is similar to that of a message with a source flowsystem that creates it (circle 1 in the figure), and hosts that process and duplicate it. In Fig. 2, the curved arrow (3) indicates the flow of the meme to many other hosts (flowsystems).

The meme is received, processed, and replicated (stored cylinder shape, circle 4) in the received state or during the process stage and may then flow to other hosts. Here, replication assumes not creating a new meme different from the original, which is not always the case, as will be discussed later. Fig. 2 represents more than copying and passing along, but also affecting (5) the meme's host.

In the Fig. 2 representation, the flow of the meme to the infected hosts is not necessarily linear (from one to another); rather, it is understood as a fanned-out flow (one-to-many). Also, the meme is replicated in the Receive and Process stages to indicate the possibility of replication of the original received meme or a modified version after processing; e.g., when an idea is passed from one individual to another, not all the exact details are replicated.

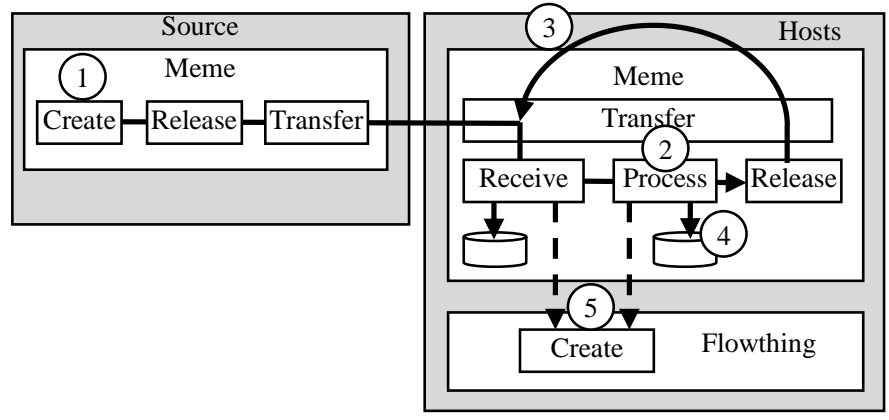

Fig. 2. A meme schema in FM

In this case the FM schema (the totality of the FM diagram) is analogous to a city map that shows all possible streets of traffic flow. Note that, as there is a source that creates a meme, its dissemination is analogous to the flow of a river through a basin as it forms a delta; eventually, memes dry out where flowsystems de-create them. This de-creation is not shown in Fig. 2 but will be shown later. Also, the lumping together of memes to form a complex meme is not shown in the figure.

Additionally, it is typically said that a meme "infection" influences a host's ideas, beliefs, behavior, etc. Hence the receiving or processing of a meme triggers (circle 5) the creation of some flowthing (e.g., an action) in the host.

Example: According to Brodie [23], a meme is a strategy, a rule of thumb for handling a situation in order to achieve some result. Typical strategy-memes in driving behavior: When you get to a traffic circle, go counter-clockwise. When you come to a red light ..., If it is green..., etc. Fig. 3 shows the flow of driving-strategy memes passing through the sphere of a Driver. Fig. 4 shows an instance of activation of the strategy when a certain traffic-light meme flows to the driver.

As mentioned in the introduction, a typical dictionary definition of evolution describes it as a process of continuous change of something into a different and usually more complex or new form. In FM, this change arises from streams of flow 
(flows across flowsystems). In Fig. 5, a meme can be changed in any host during the processing stage, but this change does not create a new meme; however, with this continuous change it is possible that a new meme can be created along the flowstream, as shown in the right-hand flowsystem of Fig. 5. Note that this flowsystem includes the Create stage.

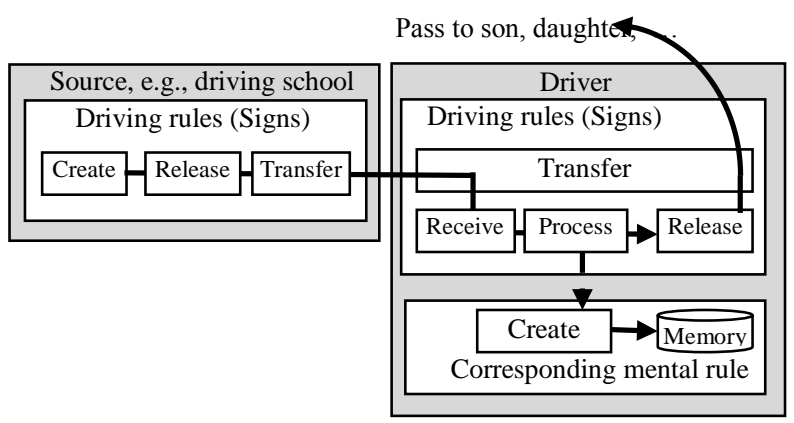

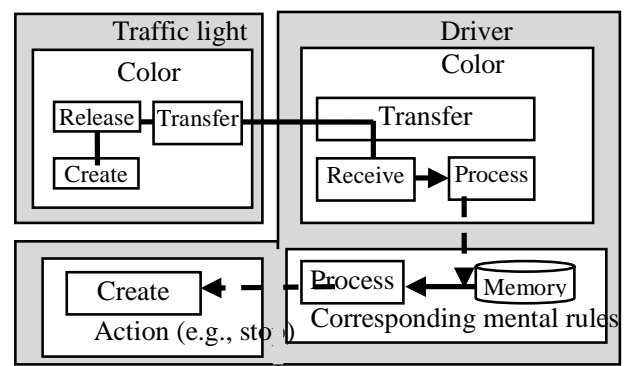

Fig. 4. Traffic light and driver's reaction

Nevertheless, it seems that other types of schemata of memes exist besides the known general form of flow of memes, as described in the next section.

Fig. 3. Example of a meme schema in FM

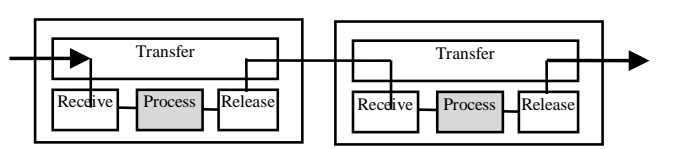

Fig. 5. Evolution of a meme

\section{APPLYING FM TO SAMPLE MEME SYSTEMS}

This section recasts four applications of memes in terms of FM. The purpose is to demonstrate that FM can uniformly express diverse situations involving memes.

\section{A. Organizational Strategy}

Speel [8] developed a description of a class of memetic evolution of an organizational strategy to achieve goals, with the aim of showing how such evolution can be described by memetics and illustrate conceptual evolution in science. According to Speel [8], a strategy is a "program of actions to be taken in order to achieve agreed upon goals" [italics added]. It is a plan to change things to solve particular problems, by defining goals to be solved and viewed as a meme-complex that evolves by selective processes, including evolutionary learning. It is assumed that an organization creates a new version of its strategy through changes in goals, and actions. The actions are connected to the goals by knowledge. New knowledge can be acquired in a kind of evolutionary learning that can take place. The evolution of a strategy is a process encompassing various players involved in different selective events. For example, these players could be categorized as having high (e.g., high-level executives), middle (e.g., middle management), or low-level judgment responsibility. [8]

Fig. 6 shows an illustration of the flows and triggering involved in the organizational strategy under consideration. A strategy is developed and flows to various units of the organization to create actions that trigger feedback used to create a new version of the strategy. The strategy is a meme that flows to different organizational units and triggers them to do actions. The units also have subunits, and the strategy flows to these subunits to trigger sub-actions.

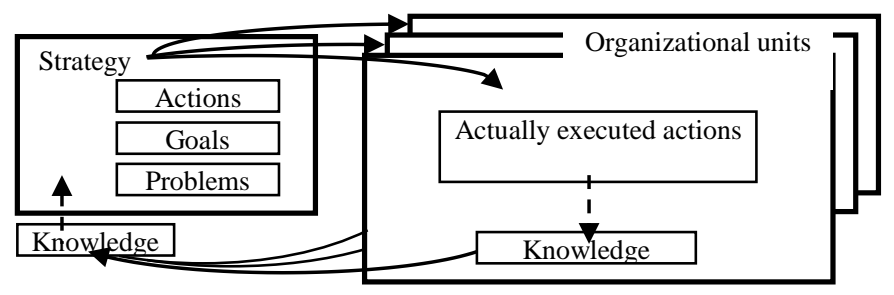

Fig. 6. Illustration of flows and triggering in an organization

It is assumed that a strategy team is responsible for developing and maintaining different versions of the strategy. In Fig. 7,

- The processing of knowledge (circle 1), problems (circle 2), goals (3), and prescribed actions (4) triggers the creation of a strategy (5).

- The processing of knowledge (6) and problems (7) triggers the creation of goals (8).

- The processing of goals (9) triggers the creation of prescribed actions

The thick vertical bar denotes a logical join. Knowledge, problems, goals, actions, and the entire strategy are flowthings that can be created, released, transferred, received, and processed. The strategy is a sphere that includes the subspheres of problems, goals, actions, and its physical self. The last flowthing (physical self) is drawn without a box and includes the stages of create (5), release, and transfer (11). The difference between the Strategy sphere and the Physical Strategy is similar to the difference between a person (as a cultural unit) and his/her physical body.

The FM representation presents a complete picture (guaranteed by continuity of flows) of all changes involved: 
appearance of new flowthings, changes in stages, in spheres, .... The schematic depiction provides a unified foundation for monitoring the evolution of all memes; otherwise, the evolution is written out in English.

Fig. 7 is still based on the general life cycle of a meme discussed in Fig. 2, where a flowthing flows to a host and

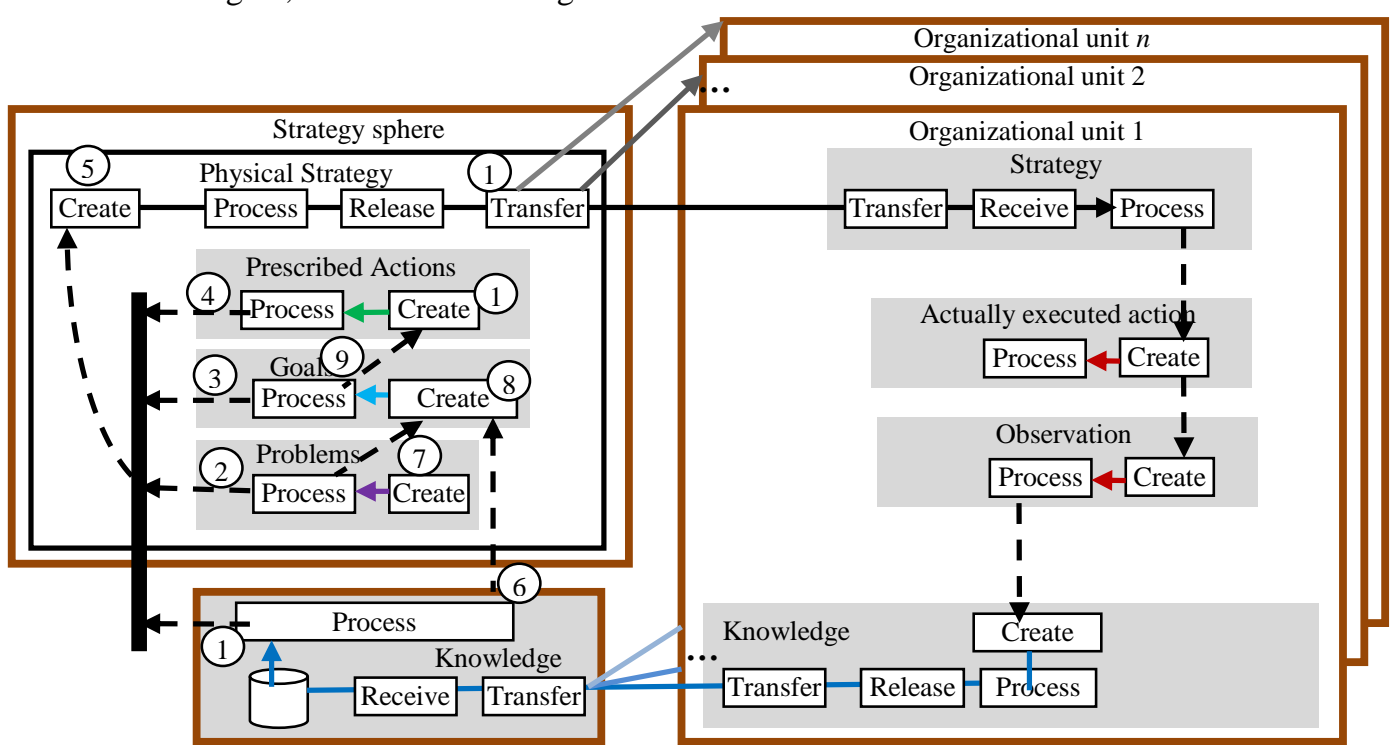

Fig. 7. FM representation of strategy flow in an organization

In terms of a meme as described by Speel [8], does strategy have the same general behavior as, say, graffiti in terms of multiplicity and spreading? Answering this type of question is not the aim of this paper. The paper presents a unifying language (FM diagrams) in which all types of "claimed" memes can be expressed and their general characteristics observed.

\section{B. Human-Centered Models of Processes}

According to Noessel [24],

Have you ever been in a design review where instead of talking about the proposed solution you spend half the time revisiting what the user is trying to accomplish in the first place? Keeping the human-centered models of the processes that lie behind your solution fresh in the minds of stakeholders (and designers) can prevent this unwanted rehashing. One way to ensure this is to create a diagram and give it qualities that make it simple enough and memorable enough so that, on a dime, you can whip out a dry-erase pen and sketch it out as a reminder...

So what about the form of the diagram? Answering this question takes us into the heady world of memes and memetics, but we'll dip just enough to come back with some meaningful attributes... A meme can be informally defined as an information structure that replicates between human minds. Examples include ... simple business process diagrams. [Italics added]

Noessel [24] gives an example of development of service for a company that supplies fire extinguishers. Six phases of the consumer product life cycle are identified, as follows (Fig. 8): affects that host (executes actions). Duplication occurs in terms of making copies available to all organizational units; however, this method of transmission does not seem to align with the popular conception of memes that spread like viruses.

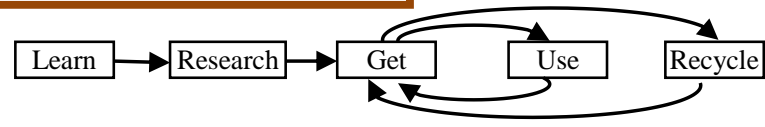

Fig. 8. Business process in a company that supplies fire extinguishers (redrawn from Noessel [24])

- Learning the need for the product

- Researching

- Acquiring the product

- If there is a fire, using the product (leading to the need for another)

- Learning when it expires (leading to the need to recycle it)

- Recycling the product when its chemicals expire (leading to need for another)

Fig. 9 shows the FM representation that corresponds to this life cycle, according to our understanding of Noessel's [24] description.

First, learning and research artifacts are received and processed (circles 1 and 2 in the figure). This triggers knowledge (circle 3). The thick vertical bar (4) indicates a joint operation. Note that this has been drawn as such for the sake of simplicity; however, it itself can be drawn as an FM diagram. Knowledge triggers the creation (5) of orders that, in turn, trigger (6) the production of fire extinguishers. Fire extinguishers flow to the utilization flowsystem (7), where they are categorized and processed as follows:

- Used, hence trigger new orders to produce more fire extinguishers (8) 
- Expired, hence they flow to recycling (9), where they are refilled with chemicals, then flow back to be utilized (10).

Note that Order in Fig. 9 has been added to the FM description even though it was not mentioned in Noessel's (2008) original scenario, where in Graph 8 Learn and Research are followed, abruptly, by Get. In FM, the notion of flow ties events together in a continuity of narration: Learn and Research trigger Knowledge, which triggers sending Order to Production of fire extinguishers.

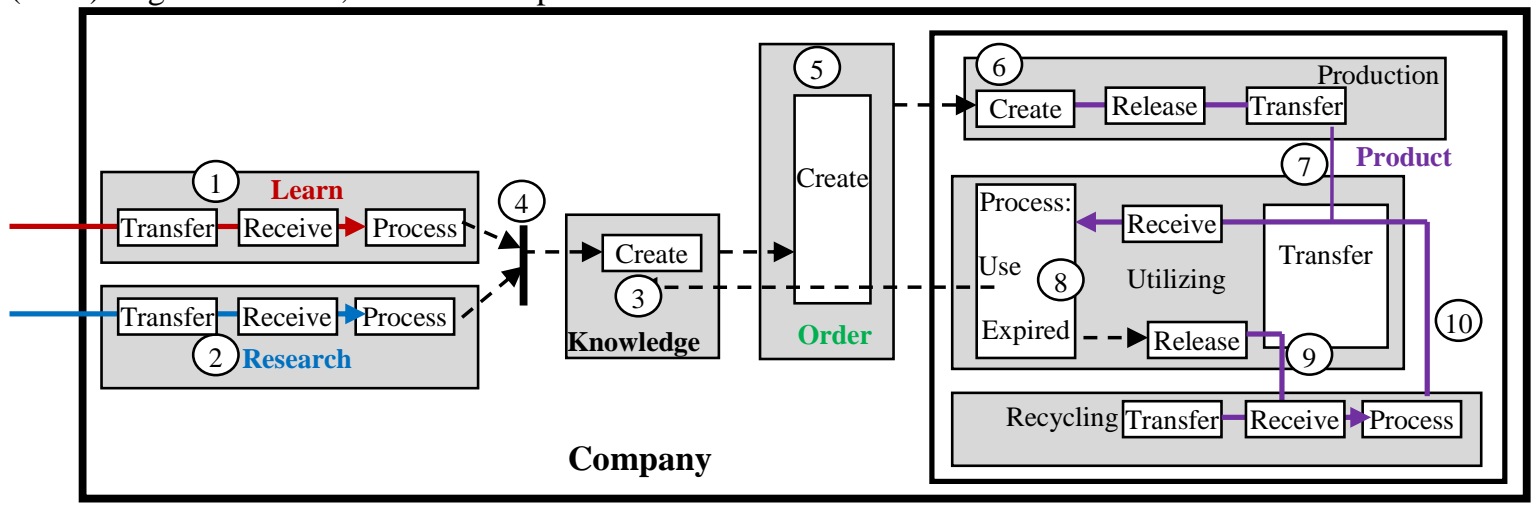

Fig. 9. FM representation of a business process for a company that supplies fire extinguishers

What characteristic of such a notion, of a "human-centered model of processes," qualifies it to take us "into the heady world of memes and memetics"? [24]. Further research can throw light on this question; what we have achieved here is the expression of human-centered models of processes while using the same diagrammatic representation for other senses of memes.

\section{Agents' Decisions about Meme Adoption and \\ Transmission}

Castelfranchi [25] studied agents' decisions with regard to meme adoption and transmission in terms of observed behaviors and actual communication. According to Castelfranchi [25],

- The so called 'contagion' can be the result of decision processes (to believe or not; to adopt or not), and

- The so called spreading can be the result of another possible decision: to pass or not to pass such a meme to others.

The agent is very far from being a passive 'vehicle' of memes; it can actively decide about receiving them and passing them. [25]

Castelfranchi [25] provides a simple model (Fig. 10) of an agent's decisions, using explicit messages aimed at inducing the agent to believe or to act. The agent's decision process is based either on observation or on communication with a source. The agent has to decide,

- whether or not to adopt such a belief, behavior, or method/tool ...

- whether or not to pass this meme on to other agents...

- or to actively try to conceal it...

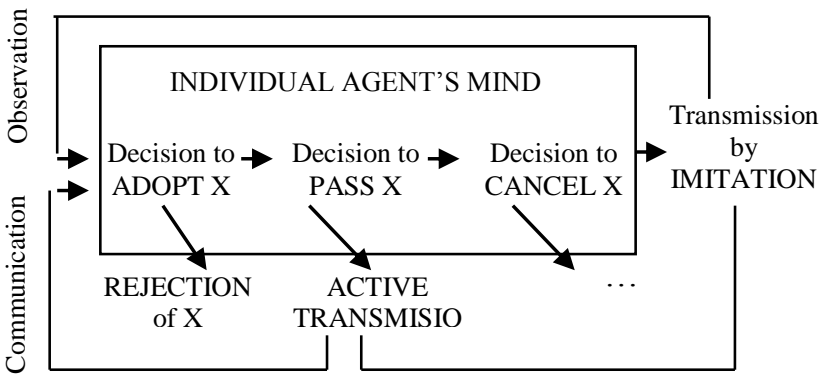

Fig. 10. Partial view of the agent's decision process (redrawn from Castelfranchi [25])

Fig. 11 shows the corresponding FM representation, according to our understanding of the description provided by Castelfranchi [25]. Memes flow from observation (circle 1) and communication (2). Note that the meme sphere in Fig. 11 comprises a hierarchy of three flowsystems, as shown in Fig. 12. Thus, a communication meme "enters" the agent sphere as such, but it can simultaneously be just a meme, the same way positive integers and negative integers can be viewed as just integers.

To emphasize this hierarchy, the meme flowsystem that includes the stages of process (3), transfer (4), receive (5), and de-create (previously called create - to be discussed later) is not drawn in a separate rectangle, as if looking upon it from above (see Fig. 13).

When the meme is processed (3), it triggers (7) the creation of a decision in the flowsystem of decisions as follows:

- "Pass" (8) triggers (9) releasing (10) the communication meme to the outside (11). Note that passing may include multiple instances of copying and passing that are not shown.

- "Adopt" (12) triggers a special type of process that can be called "use" (13). 
- "Cancel" (14) triggers de-creating (6) the meme. Note that in FM, creation is a type of flow from nonexistence to existence (appearance in the domain of discourse). De-creation is a flow in the opposite direction.

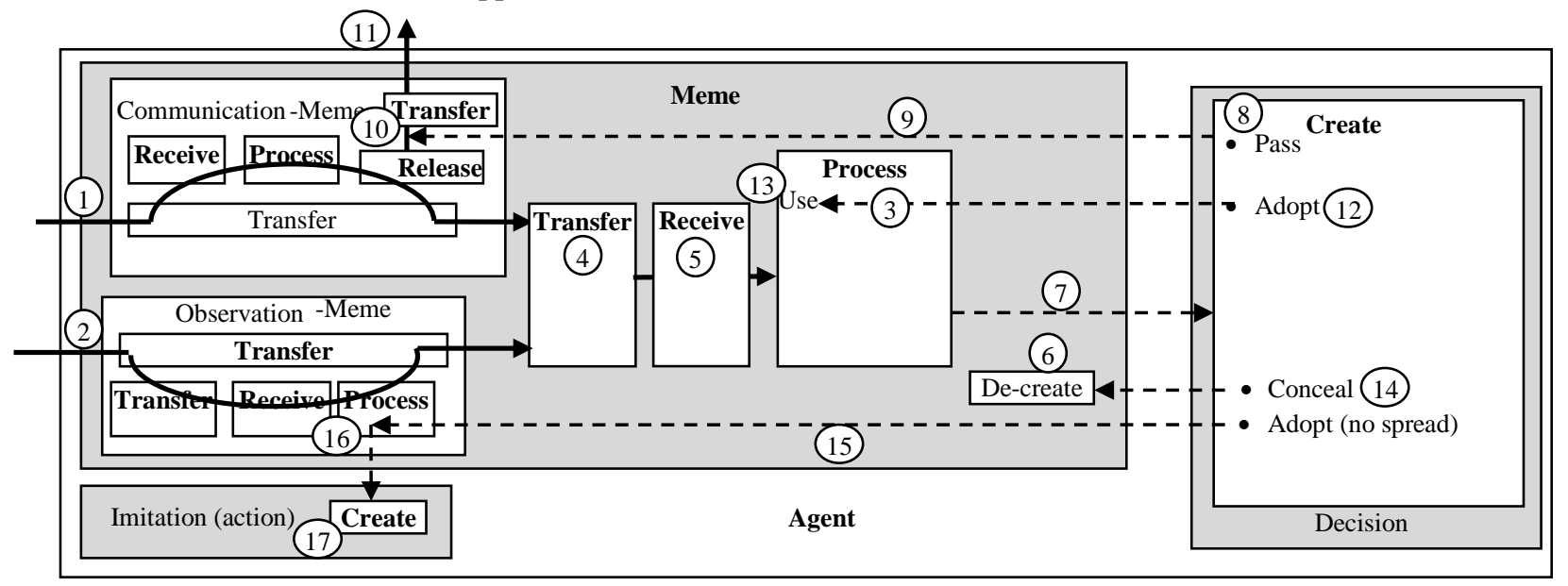

Fig. 11. FM representation of agent's decision process

- The "Adopt - no spread" of observation meme (14) triggers (15) processing of that meme (16), which in turn triggers acting the meme (17).

\section{CONCLUSION}

This paper has proposed use of the diagrammatic flowthing model (FM) as a uniform language to describe diverse conceptualizations of memes. Such uniformity paves the way in memetics to characterization and categorization of memes and to homogeneity in the field. To demonstrate the viability of this representation method, three examples were selected from different application areas and recast in FM, and the results show the method to be impressive.

The paper also provides a general FM diagram of the flow of memes from a source to infection of a host to (1) flowing to other hosts, (2) triggering some type of reaction, and (3) evolving along the stream of flow.

Future continuing research will represent more types of meme systems to identify common properties that distinguish them from diagrammatic representations of non-meme systems.

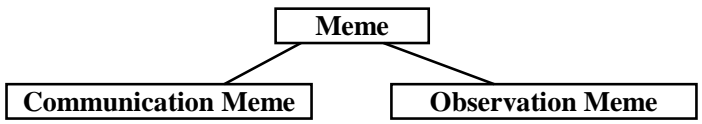

Fig. 12. A meme can be viewed in three subspheres of memes

\begin{tabular}{|l|}
\hline \multicolumn{2}{|c|}{ Meme } \\
\hline Communication Meme \\
\hline
\end{tabular}

Fig. 13. The three subspheres of memes viewed from above

\section{REFERENCES}

[1] E. Cohen, From ugly duckling to swan: Reconceptualizing information systems as a field of the discipline informing science. Journal of Computing and Information Technology, 7(3), 213-219, 1999.

[2] U. Schmitt, Putting personal knowledge management under the macroscope of informing science. Informing Science Journal, 18, 145176, 2015.
[3] T. W. Deacon, Memes as signs in the dynamic logic of semiosis: Molecular science meets computation theory, 2003. Retrieved from http://projects.chass.utoronto.ca/semiotics/srb/10-3edit.html

[4] F. Heylighen, Memetics. Principia Cybernetica Web. Nov. 23, 2001. Retrieved from http://pespmc1.vub.ac.be/memes.html

[5] R. Dawkins The selfish gene, new ed. Oxford: Oxford University Press, 1989.

[6] M. B. Prosser, Memetics - a growth industry in US military operations. MS Thesis, School of Advanced Warfighting, United States Marine Corps, 2006. 2 Retrieved www.dtic.mil/dtic/tr/fulltext/u2/a507172.pdf

[7] J. S. Wilkins, What's in a meme? Reflections from the perspective of the history and philosophy of evolutionary biology. Journal of Memetics: Evolutionary Models of Information Transmission, 2, 1998. http://cfpm.org/jom-emit/1998/vol2/wilkins_js.html

[8] H. C. Speel, A memetic analysis of policy making. Journal of Memetics: Evolutionary Models of Information Transmission, 1, 1997. Retrieved from http://cfpm.org/jom-emit/1997/vol1/speel_h-c.html

[9] I. Shifman, Memes in a digital world: Reconciling with a conceptual troublemaker. Journal of Computer-Mediated Communication, 2013. DOI: $10.1111 /$ jec 4.12013

[10] F. Heylighen, What makes a meme successful? In Proceedings, 16th International Congress on Cybernetics (Association Internat. de Cybernétique, Namur), pp. 423-418, 1998.

[11] C. Olah, Memetics [blog posting], 2011. Retrieved from https://christopherolah.files.wordpress.com/2011/01/meme.pdf

[12] H. Bjarneskans, B. Grønnevik, B. and A. Sandberg, A. The lifecycle of memes [web page], 1999. http://www.aleph.se/Trans/Cultural/Memetics/memecycle.html.

[13] G. Morgan, Images of organization. London: Sage, 1997.

[14] Heraclitus In Stanford encyclopedia of philosophy, 2011. Retrieved from http://plato.stanford.edu/entries/heraclitus

[15] D. Hollingsworth, The workflow reference model. Workflow Management Coalition (WFMC), Document No. TC00-1003, Issue 1.1, Jan. 19, 1995. Retrieved from http://www.wfmc.org/Downloaddocument/TC00-1003-The-Workflow-Reference-Model.html

[16] U. M. Husted, A funny thing happened on the way from the forum: The life and death of Internet memes. Ph.D. Thesis, University of Minnesota, 2012.

[17] S. Al-Fedaghi, Schematizing proofs based on flow of truth values in logic. IEEE International Conference on Systems, Man, and Cybernetics (IEEE SMC 2013), October 13-16, 2013, Manchester, UK.

[18] S. Al-Fedaghi, S. Flow-based enterprise process modeling. Internatonal Journal of Database Theory and Application, 6(3), 59-70, 2013. 
[19] S. Al-Fedaghi, A method for modeling and facilitating understanding of user requirements in software development. Journal of Next Generation Information Technology, 4(3), 30-38, 2013.

[20] S. Al-Fedaghi, Conceptualization of various and conflicting notions of information. Informing Science Journal, 17, 295-308, 2014.

[21] S. Al-Fedaghi, "Flow-based specification of time design requirements," Int. J. Adv. Comput. Sci. Appl. (IJACSA), vol. 6, no. 8, 2015.

[22] S. Al-Fedaghi, "Diagrammatic representation as a tool in clarifying logical arguments," Int. J. Adv. Res. Artif. Intell. (IJARAI), vol. 4, no. $10,2015$.
[23] R. Brodie, Virus and the mind: The new science of the meme. Hay House Publishers, 2009. ISBN 978-1-84850-127-0. http://media.evolveconsciousness.org/books/consciousness/Virus-of-theMind-The-New-Science-of-the-Meme-Richard-Brodie.pdf

[24] C. Noessel, Whiteboardability: How to make process diagrams memorable. Cooper Journal, May 15, 2008. Retrieved from http://www.cooper.com/journal/2008/05/whiteboardability_portraying_h

[25] C. Castlefranci, Towards a cognitive memetics: Socio-cognitive mechanisms for memes selection and spreading. Journal of Memetics: Evolutionary Models of Information Transmission, 5, 2001. http://cfpm.org/jom-emit/2001/vol5/castelfranchi_c.html 